\title{
Influence of carrier concentration on properties of InAs waveguide layers in interband cascade laser structures
}

\author{
M. Dyksik ${ }^{1}$, M. Motyka ${ }^{1}$, G. Sęk ${ }^{1}$, J. Misiewicz ${ }^{1}$, M. Dallner ${ }^{2}$, S. Höfling ${ }^{2,3}$, M. Kamp ${ }^{2}$ \\ ${ }^{1}$ Laboratory for Optical Spectroscopy of Nanostructures, Division of Experimental Physics, \\ Faculty of Fundamental Problems of Technology, Wroctaw University of Science and \\ Technology, Wybrzeże Wyspiańskiego 27, Wrocław 50-370, Poland \\ ${ }^{2}$ Technische Physik, University of Würzburg, Wilhelm-Conrad-Röntgen-Research Center \\ for Complex Material Systems, Am Hubland, Würzburg D-97074, Germany \\ ${ }^{3}$ School of Physics and Astronomy, University of St. Andrews, \\ North Haugh, St. Andrews KY16 9SS, United Kingdom
}

We present a characterization of doped InAs layers in interband cascade lasers exploiting the plasmon-enhanced waveguiding. Fast differential reflectance was employed in order to identify the plasma-edge frequency via the Berreman effect, and shown as advantageous method when compared to other types of measurements. The carrier concentration was then derived and compared with the nominal doping densities. The emission properties of the investigated structures were studied by means of photoluminescence (PL). Its full width at half maximum and integrated intensity were extracted from PL spectra and analyzed in function of the doping (carrier concentration) density. The PL linewidth was found to be independent of the carrier concentration indicating on insignificant contribution of doping to the structural properties deterioration. The PL intensity decay with the carrier concentration suggests being dominated by Auger recombination losses. 


\section{Introduction}

It is almost two decades after presentation of the basic concept of an interband cascade laser (ICL) $)^{1,2}$ and there is still a demand for further improvements of the device performance. Over the years two designs have been favored, based on gallium antimonide ( $\mathrm{GaSb}$ ) or indium arsenide (InAs) substrates. Both structures employ a broken gap alignment as an active region and the aluminum antimonide (AlSb) layer as a barrier for better carrier confinement. The typical candidate for the active region of ICLs is the so-called "W"-shaped type II quantum well made of InAs and GaInSb to confine electrons and holes, respectively. Such an approach provides the effective band gap reduction and reduces the non-radiative Auger recombination. At the same time, the spatial overlap of electron and hole wave functions is reduced compared to type I structures, having a negative impact on the oscillator strength of optical transitions and on the gain to loss ratio. Although ICLs based on GaSb substrates reached exceptional operating parameters, i.e. low threshold currents and small electrical power consumption ${ }^{3,4}$, operation far above room temperature ${ }^{5,6}$ in the $3-5.5 \mu \mathrm{m}$ range, several active region's modifications have been studied in order to tailor the emission wavelength deeper into the mid-infrared and to provide enhancement for the oscillator strength of the active optical transition: i) increasing the InAs layer width ${ }^{7}$; ii) incorporation of arsenic atoms into GaInSb resulting in a quaternary GaInAsSb layer for the confinement of holes ${ }^{8,9}$; iii) modification of the standard W-shaped InAs/GaInSb/InAs quantum well by application of additional InAs and GaInSb layers resulting in a multiple quantum well (QW) design, ${ }^{10,11}$ iv) and replacement of GaInSb with a GaAsSb ternary alloy ${ }^{12}$.

All above-listed modifications regard mainly GaSb-based structures. Due to the lack of lattice-matched materials with a high contrast of refractive indices on $\mathrm{GaSb}$, these structures utilize a strain balanced InAs/AlSb superlattice (SL) as cladding layer, which has a lower effective refractive index than the GaSb separate confinement layers surrounding the active region. However, the molecular beam epitaxy of these InAs-AlSb SL claddings is very demanding, because the SL cladding layer is several microns thick and usually has more than 2000 interfaces $^{13}$. This requires constant shutter switching during the growth process and makes it hard to achieve a high-quality strain-balanced material. Furthermore, longer modal characteristic decay lengths and stronger absorption in the spectral region beyond $6 \mu \mathrm{m}$ would require even thicker SL cladding layers as compensation, leading to higher thermal resistance, poorer heat dissipation and even longer growth times. These challenges make the SL approach rather unsuitable at longer wavelengths.

An already reported, the possible modification of the ICL structure is to employ a 
quaternary AlGaAsSb bulk cladding layer ${ }^{14}$, or highly doped $n^{+}$-InAs layer as a plasmonenhanced waveguide. Recently Lu Li et al. demonstrated an InAs-based ICL with plasmonenhanced waveguide lasing at the record wavelength around $11 \mu \mathrm{m}$ in pulsed mode up to 130 $\mathrm{K}^{15}$. On the other hand, the group from the University of Würzburg coauthoring the current communication presented an ICL grown on an InAs substrate lasing at the record wavelength of $7 \mu \mathrm{m}$ at room temperature in pulsed mode ${ }^{16}$, with threshold current densities as low as 800 $\mathrm{A} / \mathrm{cm}^{2}$. Although the obtained threshold current densities of the InAs-based ICLs are remarkably low taking into account the immaturity of the InAs technology, there is still a gap to state-of-the-art GaSb-based structures, with the room temperature threshold current densities below $100 \mathrm{~A} / \mathrm{cm}^{2}$ at a wavelength of $3.6 \mu \mathrm{m}$.

As the highly doped $\mathrm{n}^{+}$-InAs cladding layer has a thickness of several microns, the structure is grown lattice matched on an InAs substrate, thereby greatly simplifying the epitaxial growth. Also the thermal resistance is lower in the case of doped InAs when compared to InAs/AlSb superlattices, leading to better thermal conductivity, thus addressing the key problem in the case of the superlattice-based waveguide. What is more, the thermal conductivity changes rather weakly in the range of doping concentrations of $10^{16}-10^{18} \mathrm{~cm}^{-3},{ }^{17}$ which is beneficial in case of $\mathrm{n}^{+}$-InAs ICLs claddings allowing for their engineering without deteriorating the thermal conductivity.

The effective optical mode confinement inside the cascade stage might be described by the optical mode penetration ( $\left.\Gamma_{\text {cladd }}\right)$ into the cladding region. As reported previously in Ref. 13, the common approach of a InAs-AlSb SL exhibits a $\Gamma_{\text {cladd }}$ of 57\%, whilst a similar device with a plasmon-enhanced waveguide shows a $\Gamma_{\text {cladd }}$ of $4 \%$. In addition, the waveguides with a highly doped $\mathrm{n}^{+}$-InAs layer benefit from the plasmon-enhanced reflection - each frequency below the plasma-edge frequency is consistently reflected and therefore enhanced inside the active medium. Also the refractive index is reduced in case of doped InAs claddings, thus providing better optical mode confinement inside the active region as a result of higher contrast between respective refractive indices. On the other hand, the high level of dopants enhances the free carrier absorption, influencing the optical losses and affecting the gain and the threshold current densities. Therefore, the doping level in the InAs cladding layers has to be chosen carefully in order to minimize these mentioned losses and, at the same time, to provide good optical mode confinement. Hence, a precise design and experimental verification of the carrier concentration in the plasmon-enhanced waveguide is crucial for the optimization of the abovementioned factors.

There is a limited number of methods for measuring carrier concentration in bulk 
semiconductors or low-dimensional semiconductor structures. The Hall and capacity-voltage measurements are at forefront, despite their destructive nature i.e. the sample is no longer suitable to process into a laser. Both methods require an additional sample preparation, including fabrication of electrical contacts and, in the case of Hall measurement, an external magnetic field. This makes the electrical-based approaches destructive and time consuming.

The aim of this work is to perform quantitative analysis of the doping concentration in the plasmon-enhanced waveguides of ICLs by non-destructive and fast methods of optical spectroscopy. In contrast to other reports in this field, we employed an absorption-like differential technique: Fast Differential Reflectance (FDR) to realize this goal. Although the differential reflectivity techniques are very well established for characterization of semiconducting materials, low-dimensional structures and devices, it has not been used to detect the plasma frequencies and hence to determine the carrier concentrations, which we propose here. The obtained differential-like spectra lie on a zero baseline with no evidence of features related to the set-up characteristic and/or the environmental conditions the measurement are taken at. The above factors allow for precise selection of the plasma-edge frequency, in contrast to the reflectance measurements where the respective minimum is usually located among other features. Our approach simplifies the analysis of usually complex mid-infrared spectra, therefore, it can be advantageous in case of at least some kinds of heterostructures. In addition to that, the emission properties of the investigated structures are also tested by means of mid-infrared photoluminescence spectroscopy. The impact of doping densities on the full width at half maximum and the integrated intensity of the PL signal are discussed.

\section{Structures and methods}

Two sets of structures were grown in a solid-source molecular beam epitaxy system equipped with valved cracker cells for both antimony and arsenic. The first one consisted of three samples with different nominal carrier concentrations $\left(2 \times 10^{17}, 2 \times 10^{18}\right.$ and $\left.2 \times 10^{19} \mathrm{~cm}^{-3}\right)$ in $200 \mathrm{~nm}$ thin Si-doped InAs layers and was grown for the purpose of reference. The second one included interband cascade lasers with $1.6 \mu \mathrm{m}$ thick Si-doped InAs cladding layers, with the nominal carrier concentrations in the plasmon-enhanced waveguide varied in the range of $5 \cdot 10^{18}-8 \cdot 10^{19} \mathrm{~cm}^{-3}$. The Si-doped InAs layers in the first sample set were grown at a rate of $500 \mathrm{~nm} / \mathrm{h}$. For the thick InAs layers in the second set a higher growth rate of $850 \mathrm{~nm} / \mathrm{h}$ was used. In both cases the InAs layers were grown at a substrate temperature of $450{ }^{\circ} \mathrm{C}$, measured by a pyrometer. The $\mathrm{As}_{2} /$ In flux ratio was kept in the range of $10-20$ for all InAs 
layers. The SIMS measurements on Si-doped InAs samples were used to calibrate the Si flux. $\mathrm{Si}$ atoms were counted and it was assumed it would roughly equal the actual carrier concentration.

In order to determine the plasma frequency and hence to calculate the carrier concentration in both the highly-doped InAs layers and the ICL waveguides, the Berreman effect $^{18}$ was employed. The Berreman effect is manifested in the oblique-incidence spectrum by an enhancement in the absorption of $p$-polarized light in the vicinity of the plasma-edge. The origin of this minimum is understood as a coupled plasmon-phonon mode, usually visible in the spectra of Raman spectroscopy. In this report, we present a novel approach to plasmaedge frequency determination based on analysis of an FDR spectrum. Although our approach is consistent with the previously presented methods ${ }^{19}$, a number of significant advantages resulting from the differential nature of the FDR measurement will be presented in the subsequent paragraph, including a straightforward plasma-edge frequency determination due to the zero baseline and elimination of the thermal background signal and the spectral set-up characteristics.

In this report results of two independent techniques of optical spectroscopy are discussed, namely the FDR and PL. Both were realized with a setup based on a Fourier transform infrared spectrometer Bruker 80v. A Mercury Cadmium Telluride photodetector and a Si bolometer were used in order to cover the spectral regions of $5-15 \mu \mathrm{m}$ and $15-50$ $\mu \mathrm{m}$, respectively.

The FDR measurements were performed in the rapid-scan mode, with a spectral resolution of $2 \mathrm{~cm}^{-1}$. An external chamber was utilized providing an oblique incidence of 45 degrees for the probe light. The globar light was reflected off the sample and focused on the detector with a set of parabolic mirrors. For each sample two spectra, with and without a pump laser beam, were measured and analyzed by external software. The pump beam was provided by a $661 \mathrm{~nm} 40 \mathrm{~mW}$ continuous-wave semiconductor laser diode. For more information about the FDR technique and its differential nature the reader is referred to previous works ${ }^{20,21}$.

The PL measurements were performed in a standard step-scan Fourier transform infrared spectroscopy configuration, with phase sensitive detection made by a lock-in amplifier. The same pump beam was mechanically chopped at a reference frequency of 280 $\mathrm{Hz}$ and focused onto the sample to a spot of $1 \mathrm{~mm}^{2}$. The spectra were measured with the resolution of $64 \mathrm{~cm}^{-1}$ and the signal on the detector was averaged over time in order to improve the signal to noise ratio. To realize that, a standard procedure was employed where 
the spectrometer's scanning mirror was fixed at each position for $500 \mathrm{~ms}$.

\section{Results and discussion}

Figure 1a presents the reflection spectrum of a highly doped InAs thin film measured at the angle of incidence of 45 degrees. It has been previously presented by Hinkey et al. ${ }^{19}$ that reflectance measurements are suitable for the determination of plasma frequency in highly doped InAs thin films. However, the spectrum in Fig. 1a contains several minima and some of them are related to absorption by ambient gasses or the set-up characteristic (marked with vertical dashed arrows), making the interpretation of the spectra ambiguous and a misinterpretation possible. Figure $1 \mathrm{~b}$ presents the corresponding FDR spectrum. Clearly, the abovementioned advantages of the FDR approach are visible. The spectrum contains only two singularities in the dielectric response of the investigated structure, including the "plasmaedge frequency" feature at $145 \mathrm{meV}$ and an optical transition at $\sim 350 \mathrm{meV}$ understood as related to the InAs substrate bandgap. The spectrum lies well on a zero baseline, which greatly simplifies the determination of the plasma-edge frequency. Furthermore, the background signal related to the set-up characteristic and the absorption by ambient gasses is consecutively removed due to the differential nature of the FDR measurement. In order to prove that the singularity at a low-energy side corresponds to the Berreman minimum in the reflectance measurement, the FDR spectra were measured for two orthogonal polarizations and presented in Figure 1c. For the p-polarized light of incidence (red dotted line) a singularity at the frequency corresponding to the plasma-edge is visible, while for the blue dashed curve the spectrum is flat.

Since the measured singularity energy, attributed to the plasma-edge, allows for calculations of carrier concentration, it is worth mentioning that Fig. $1 \mathrm{~b}$ provides the necessary information even for non-polarized light of incidence. This lifts the requirements for a polarizer, which is generally beneficial in the case of mid- and far-infrared spectroscopy, especially when various frequencies/wavelengths in a broad spectral range (range of concentrations) are considered.

This confirmed observation of the plasma-edge features in the differential reflectivity spectra under the laser modulation suggest also on the most probable mechanism of this photomodulation. This is a small change in the carrier concentration (due to laser-related photo-generated carriers) and the related modification of the refractive index. The latter causes a non-zero difference between the two reflectivity spectra being subtracted, and giving rise to a singularity in $\Delta \mathrm{R}$. 
As mentioned above, the knowledge of the plasma-edge frequency allows for calculations of the carrier concentration. The plasma frequency is defined as:

$$
\omega_{p}^{2}=\frac{n e^{2}}{\varepsilon_{0} \varepsilon_{\infty} m^{*}}
$$

where $\varepsilon_{\infty}$ is the high frequency limit of the dielectric constant $\left(11.7\right.$ for $\left.\operatorname{In} A s^{22}\right)$ and $\varepsilon_{0}$ is the permittivity of free space. Since the investigated structures are highly-doped and the band structure of InAs is highly non-parabolic away from the $\Gamma$ zone center, it is crucial to include the electron effective mass dependence on the carrier concentration $m^{*}(n)$. For a spherical band the following equation is fulfilled: ${ }^{23}$

$$
m^{*}(n)=m_{e}\left[1+\frac{4 P^{2}}{3 E_{g}}\left(1+\frac{8 P^{2} \hbar^{2}\left(3 \pi^{2} n\right)^{\frac{2}{3}}}{3 m_{e} E_{g}^{2}}\right)^{-\frac{1}{2}}\right]^{-1}
$$

where $P^{2}$ is the momentum matrix element of the coupling between conduction and valence bands and is assumed to be $11.9 \mathrm{eV}^{23}$ and $m_{e}$ is the electron rest mass. All the parameters required for the calculations are taken from Ref. 24.

Figure 2 presents the FDR results measured for the three ICLs with different nominal carrier concentrations in the InAs plasmonic waveguide. Even though the spectra in Fig. 2 are more complicated than in case of the InAs thin films, the singularity corresponding to the plasma-edge frequency is still straightforwardly appointed. For each measured sample in the case of $p$-polarized light of incidence (red dotted line) one may distinguish a spectral feature marked with the dashed arrow. Alike the case of InAs thin films (Fig. 1c), the blue dashed curves attributed to the orthogonal polarization are flat in the vicinity of plasma edge frequency. One can notice that for frequencies above the plasma frequency, for all the investigated laser structures, a region with additional non-polarized features is present and attributed to the Fabry-Perot cavity modes. This specific behavior of the Fabry-Perot cavity modes allows the direct identification of singularities being polarization dependent i.e. singularities of longitudinal optical phonon origin (plasma frequency) in the polarizationresolved FDR measurement of a complicated multilayer structure.

Figure 3 summarizes all the measured values of the plasma-edge frequencies with the 
calculated values of carrier concentration. Each point is labeled with the nominal carrier concentration. The discrepancy between the calculated and nominal values is due to the fact that the optical methods of carrier concentration determination are sensitive only to the $\mathrm{Si}$ atoms that act as donors and contribute to the free electron density, while the nominal concentration assumed during the growth procedure also includes the dopants playing the role of both substituent of arsenic atoms and interstitials in the lattice. For the low-doped InAs thin film $\left(2 \times 10^{17} \mathrm{~cm}^{-3}\right)$ the calculated plasma-edge frequency lies in the vicinity of the longitudinal optical phonon mode thus the accurate frequency of the plasma-edge cannot be extracted.

In indium arsenide the electron effective mass dependence on carrier concentration cannot be neglected, especially when the doping densities exceed $1 * 10^{18} \mathrm{~cm}^{-3}$. For the carrier concentration of $1 * 10^{18} \mathrm{~cm}^{-3}$ the $\mathrm{m} *(\mathrm{n}) / \mathrm{m}_{\mathrm{e}}$ ratio equals to 0.036 and increases to 0.064 for carrier density of $1 * 10^{19} \mathrm{~cm}^{-3}$. This situation is presented in Fig. 3 and manifested by different slopes of the dashed and dotted lines. The light dashed line stands for Eq. 1 when the electron effective mass is constant and the dotted line is for the purpose of guidance.

In order to find out if the high level of doping has any detrimental influence on the structural quality in addition to other doping-independent inhomogeneities, the photoluminescence spectra have been measured at liquid nitrogen temperature in function of the carrier concentration. The PL lineshape was analyzed by means of a Gaussian function in order to extract the linewidth and integrated PL intensity. The broadening presented in Fig. 4a remains nearly constant (approx. $16 \mathrm{meV}$ ) in this range of carrier densities: $2 \cdot 10^{17}$ $8 \cdot 10^{19} \mathrm{~cm}^{-3}$. Such behavior indicates that the high level of doping does not contribute significantly to the deterioration of the structural properties, which are apparently dominated by other factors. It is also not the thermal broadening because temperature dependence of PL (not shown here) showed that the linewidth is approximately constant in the range of low temperatures up to $100 \mathrm{~K}$. Therefore, the observed inhomogeneous linewidth is rather mostly related to intrinsic structure/material nonuniformities, which however are not significantly affected by the increasing doping. The inset to Fig. 4a presents four PL spectra corresponding to samples with different nominal carrier concentrations - the spectra are vertically shifted for clarity. The broadening of each spectrum is constant, presenting a direct evidence of the linewidth independence on the carrier/doping concentration.

Figure $4 \mathrm{~b}$ presents the integrated PL intensity from highly doped InAs of both thin films and waveguides as a function of the carrier concentration (calculated on the basis of plasma-edge frequency). Since the linewidth exhibits a constant value in the range of the discussed doping levels and the investigated samples might be considered degenerate due to 
the high level of doping, the tendency towards lower values of the integral PL intensity with an increase in the carrier concentration points at the Auger recombination as the predominant losses mechanism. Although we are not capable of direct derivation of the waveguide losses, the forthcoming analysis provides some estimation.

The integral PL intensity is proportional to the internal quantum efficiency $\eta_{\text {int }}$ On the other hand the $\eta_{\text {int }}$ is equal to the ratio of both radiative $\left(\Gamma_{\mathrm{r}}\right)$ and total $\left(\Gamma_{\mathrm{t}}\right)$ recombination processes, with the latter including Shockley-Read-Hall (SRH) and Auger mechanisms ${ }^{25}$, thus the $\eta_{\text {int }}$ might be ascribed as:

$$
\eta_{\text {int }}=\frac{\Gamma_{\mathrm{r}}}{\Gamma_{\mathrm{t}}}=\frac{A}{A+B+C n^{\beta}}
$$

where $\mathrm{A}, \mathrm{B}$ and $\mathrm{C}$ are the radiative recombination coefficient, $\mathrm{SRH}$ coefficient and Auger coefficient, respectively. At this point it is worth reminding that our studies are based on degenerate InAs where due to the high level of doping and a small InAs electron effective mass, the Fermi level is located approximately $100-400 \mathrm{meV}$ above the conduction band edge $^{19}$. It has been shown elsewhere ${ }^{25-27}$ that the inverse Auger lifetime in the case of narrowband gap and degenerate semiconductors has a linear dependence on the doping concentration $n$ (which particularly translates into $\beta=1$ in Eq. 3) and the other two coefficients, A and B, do not depend on $n$. The standard approach to determine $\mathrm{C}$ is to perform measurements in the socalled high-excitation regime, where the number of photoexcited electrons $\Delta n$ (holes $\Delta p$ ) is varied. Since our studies are based on degenerate InAs with the Fermi level far above the conduction band edge, we instead used a variation of $n$. This assumption is supported by $\Delta n$ $<<n$, which is fulfilled in our case since the estimated average number of electrons photoexcited by the pump beam is approximately $10^{15} \mathrm{~cm}^{-3}$. Although our model is greatly simplified, it shows that Eq. 3 satisfies the abovementioned criteria and gives reasonable values of $\mathrm{C}$ with previously assumed linear dependence on $n$. The radiative coefficient $\mathrm{A}$, understood as the dipole transition rate constant, is calculated to be $2.4 * 10^{8} \mathrm{~s}^{-1}$ for degenerate bulk InAs at $77 \mathrm{~K}^{28}$, whereas the SRH rate constant is expected to be low for the epitaxially grown structures and its upper limit can only be estimated here. This limit of B can be derived from the ratio of the integrated PL intensity at low and room temperatures, which equals to 0.24 for our least doped sample $\left(2 * 10^{17} \mathrm{~cm}^{-3}\right)$, resulting in a B constant of about $1 * 10^{9} \mathrm{~s}^{-1} 25$. The knowledge of A and on upper limit of B allows fitting the solid points in Fig. $4 \mathrm{~b}$ with the function from Eq. (3). As a result, the Auger coefficient $\mathrm{C}$ can be estimated as 
$4.5^{*} 10^{-10} \mathrm{~cm}^{3} \mathrm{~s}^{-1}$, corresponding to the inverse Auger lifetime of approx $10 \mathrm{~ns}$ for the concentration of $2 * 10^{17} \mathrm{~cm}^{-3}$, which is consistent with the previously presented value ${ }^{29}$. Also, the linear dependency on the doping concentration is confirmed by the fitting procedure with the parameter $\beta$ being equal to 1.03 .

\section{Conclusions}

In conclusion, we have examined highly-doped cladding layers of interband cascade lasers by the combination of two optical spectroscopy techniques: fast differential reflectance and photoluminescence. The FDR measurements provided information about the plasma frequencies and the related carrier concentration showing several advantages of this approach: (i) due to differential-like nature the spectra lie on zero baseline with very often featureless background which allows for precise detection of the plasma-edge frequency; (ii) it lifts, in some cases, the requirement for the use of a polarization optics, beneficial especially, if a broad range of concentrations (broad spectral range) is considered; (iii) it opens a path for exploitation of other differential techniques, as photoreflectance, i.e. single scan experiments; (iv) it can directly be used for other doped materials and devices, as quantum cascade lasers; (v) the simplicity of the FT-based experiment and its interpretation makes it useful for material engineers and industrial laboratories, as fast, simple, contactless and non-destructive probe of carrier concentration.

Eventually, the PL data showed no effect of the high level of doping on the linewidth whereas the PL intensity decrease with increasing carrier concentration seems to be dominated by losses due to the Auger recombination. Both the PL intensity and the internal quantum efficiency decay significantly for carrier concentrations above $10^{19} \mathrm{~cm}^{-3}$, which are, however, required in order to shift the plasma-edge frequency into the mid-infrared spectral range. Therefore, the optimized ICL design with doped claddings in that range of high carrier concentrations is very challenging, and is a compromise between the radiative properties and the proper optical mode confinement.

\section{Acknowledgements}

The work has supported by the European Commission within the Project WideLase (No. 318798) of the 7-th Framework Programme and by the National Science Center of Poland by the grant no. 2014/15/B/ST7/04663. 


\section{Figure captions}

\section{Figure 1.}

Comparison of results obtained by (a) the reflectance measurement (b) the FDR measurement and (c) the FDR for two orthogonal polarizations $p$ and $s$.

\section{Figure 2.}

Results of the FDR measurements for three ICLs with different doping densities in the InAs plasmon waveguide. The vertical dashed arrows indicate the plasma frequency.

\section{Figure 3.}

Summary of plasma-edge frequencies calculated for thin InAs films (squares) and plasmonenhanced InAs waveguides (circles). Each point is labeled with the nominal concentration. The dashed line represents the plasma-edge frequency dependence on carrier concentration with constant electron effective mass. The dotted line is a guide for the eye.

\section{Figure 4.}

PL linewidth versus carrier concentration for highly-doped cladding layers (a) and the integral PL intensity as a function of carrier concentration for all investigated structures with black solid line representing a fit with Eq. 3 (b). The inset in upper panel presents the analyzed PL spectra. 


\section{References}

${ }^{1}$ R.Q. Yang, Superlattices Microstruct. 17, 77 (1995).

2 J.R. Meyer, C.A. Hoffman, F.J. Bartoli, and L.R. Ram-Mohan, Appl. Phys. Lett. 67, 757 (1995).

${ }^{3}$ I. Vurgaftman, W.W. Bewley, C.L. Canedy, C.S. Kim, M. Kim, C.D. Merritt, J. Abell, J.R. Lindle, and J.R. Meyer, Nat. Commun. 2, 585 (2011).

${ }^{4}$ I. Vurgaftman, R. Weih, M. Kamp, J.R. Meyer, C.L. Canedy, C.S. Kim, M. Kim, W.W. Bewley, C.D. Merritt, J. Abell, and S. Höfling, J. Phys. D. Appl. Phys. 48, 123001 (2015).

${ }^{5}$ W.W. Bewley, C.S. Kim, C.L. Canedy, C.D. Merritt, I. Vurgaftman, J. Abell, J.R. Meyer, and M. Kim, Appl. Phys. Lett. 103, 10 (2013).

${ }^{6}$ C.S. Kim, M. Kim, J. Abell, W.W. Bewley, C.D. Merritt, C.L. Canedy, I. Vurgaftman, and J.R. Meyer, Appl. Phys. Lett. 101, 10 (2012).

${ }^{7}$ M. Motyka, G. Sęk, J. Misiewicz, A. Bauer, M. Dallner, S. Höfling, and A. Forchel, Appl. Phys. Express 2 , 126505 (2009).

${ }^{8}$ F. Janiak, M. Motyka, G. Sęk, M. Dyksik, K. Ryczko, J. Misiewicz, R. Weih, S. Höfling, M. Kamp, and G. Patriarche, J. Appl. Phys. 114, 223510 (2013).

${ }^{9}$ K. Ryczko, G. Sęk, and J. Misiewicz, J. Appl. Phys. 114, (2013).

${ }^{10}$ M. Motyka, K. Ryczko, M. Dyksik, G. Sęk, J. Misiewicz, R. Weih, M. Dallner, S. Höfling, and M. Kamp, J. Appl. Phys. 117, 084312 (2015).

${ }^{11}$ Y. Jiang, L. Li, Z. Tian, H. Ye, L. Zhao, R.Q. Yang, T.D. Mishima, M.B. Santos, M.B. Johnson, and K. Mansour, J. Appl. Phys. 115, (2014).

${ }^{12}$ K. Ryczko, G. Sęk, and J. Misiewicz, Appl. Phys. Express 8, 121201 (2015).

${ }^{13}$ Z. Tian, C. Chen, R.Q. Yang, T.D. Mishima, M.B. Santos, J.C. Keay, M.B. Johnson, and J.F. Klem, in Proc. SPIE 7616 (2010), p. 76161B-76161B-9.

${ }^{14}$ R. Weih, A. Bauer, M. Kamp, and S. Höfling, Opt. Mater. Express 3, 1624 (2013).

${ }^{15}$ L. Li, H. Ye, Y. Jiang, R.Q. Yang, J.C. Keay, T.D. Mishima, M.B. Santos, and M.B. Johnson, J. Cryst. Growth 425, 369 (2015).

${ }^{16}$ M. Dallner, F. Hau, S. Höfling, and M. Kamp, Appl. Phys. Lett. 106, 041108 (2015).

${ }^{17}$ R. Bowers, R.W. Ure, J.E. Bauerle, and A.J. Cornish, J. Appl. Phys. 30, 930 (1959).

${ }^{18}$ D. Berreman, Phys. Rev. 130, 2193 (1963).

${ }^{19}$ R.T. Hinkey, Z. Tian, R.Q. Yang, T.D. Mishima, and M.B. Santos, J. Appl. Phys. 110, 043113 (2011).

${ }^{20}$ M. Motyka and J. Misiewicz, Appl. Phys. Express 3, 112401 (2010).

${ }^{21}$ M. Motyka, G. Sęk, F. Janiak, J. Misiewicz, K. Kłos, and J. Piotrowski, Meas. Sci. Technol. 22, 125601 (2011). 
${ }^{22}$ E.D. Palik, Handbook of Optical Constants of Solids (Academic Press, New York, 1985).

${ }^{23}$ Y.B. Li, R.A. Stradling, T. Knight, J.R. Birch, R.H. Thomas, C.C. Phillips, and I.T. Ferguson, Semicond. Sci. Technol. 8, 101 (1993).

${ }^{24}$ I. Vurgaftman, J.R. Meyer, and L.R. Ram-Mohan, J. Appl. Phys. 89, 5815 (2001).

${ }^{25}$ Y. Cho, X. Lue, M. Wienold, M. Ramsteiner, H.T. Grahn, and O. Brandt, Phys. Rev. B 87, 155203 (2013).

${ }^{26}$ A. Haug, Solid. State. Electron. 21, 1281 (1978).

${ }^{27}$ Y.D. Jang, T.J. Badcock, D.J. Mowbray, M.S. Skolnick, J. Park, D. Lee, H.Y. Liu, M. Hopkinson, R. a Hogg, and a D. Andreev, Appl. Phys. Lett. 93, 101903 (2008).

${ }^{28}$ J.R. Lindle, J.R. Meyer, C.A. Hoffman, F.J. Bartoli, G.W. Turner, and H.K. Choi, Appl. Phys. Lett. 67, 3153 (1995)

${ }^{29}$ V.N. Abakumov, V.I. Perel, and I.N. Yassievich, in Mod. Probl. Condens. Matter Sci. (1991). 


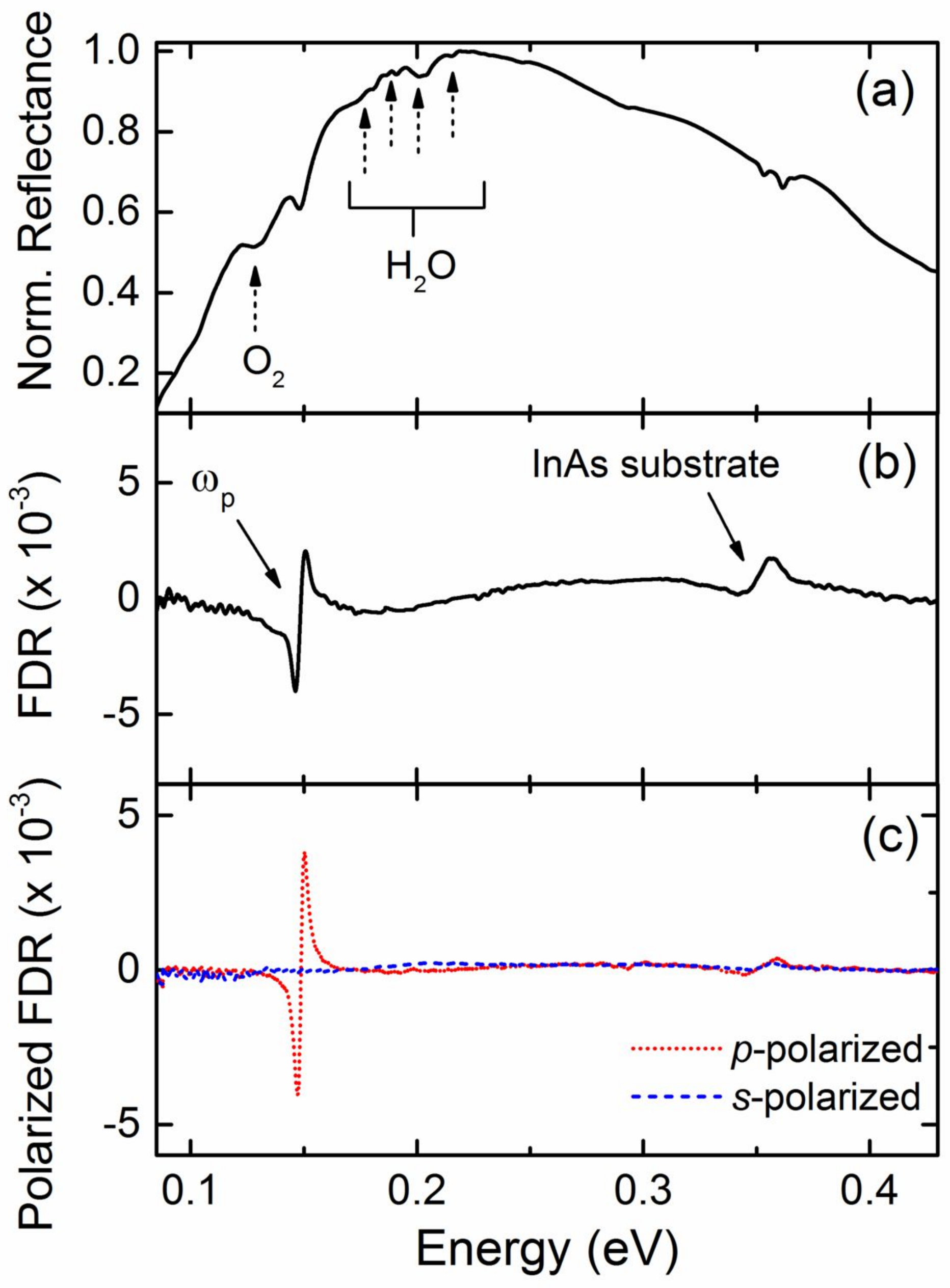




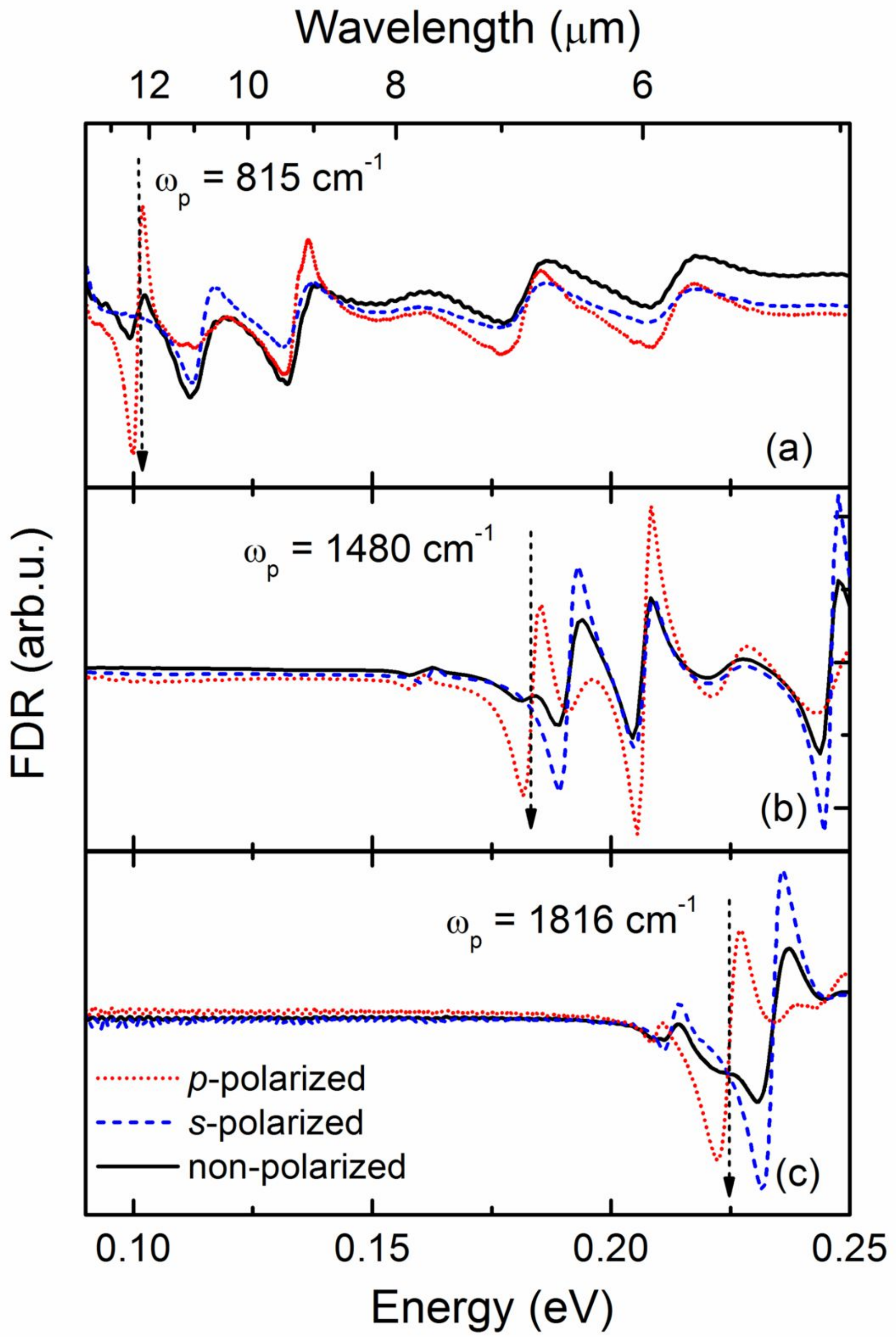




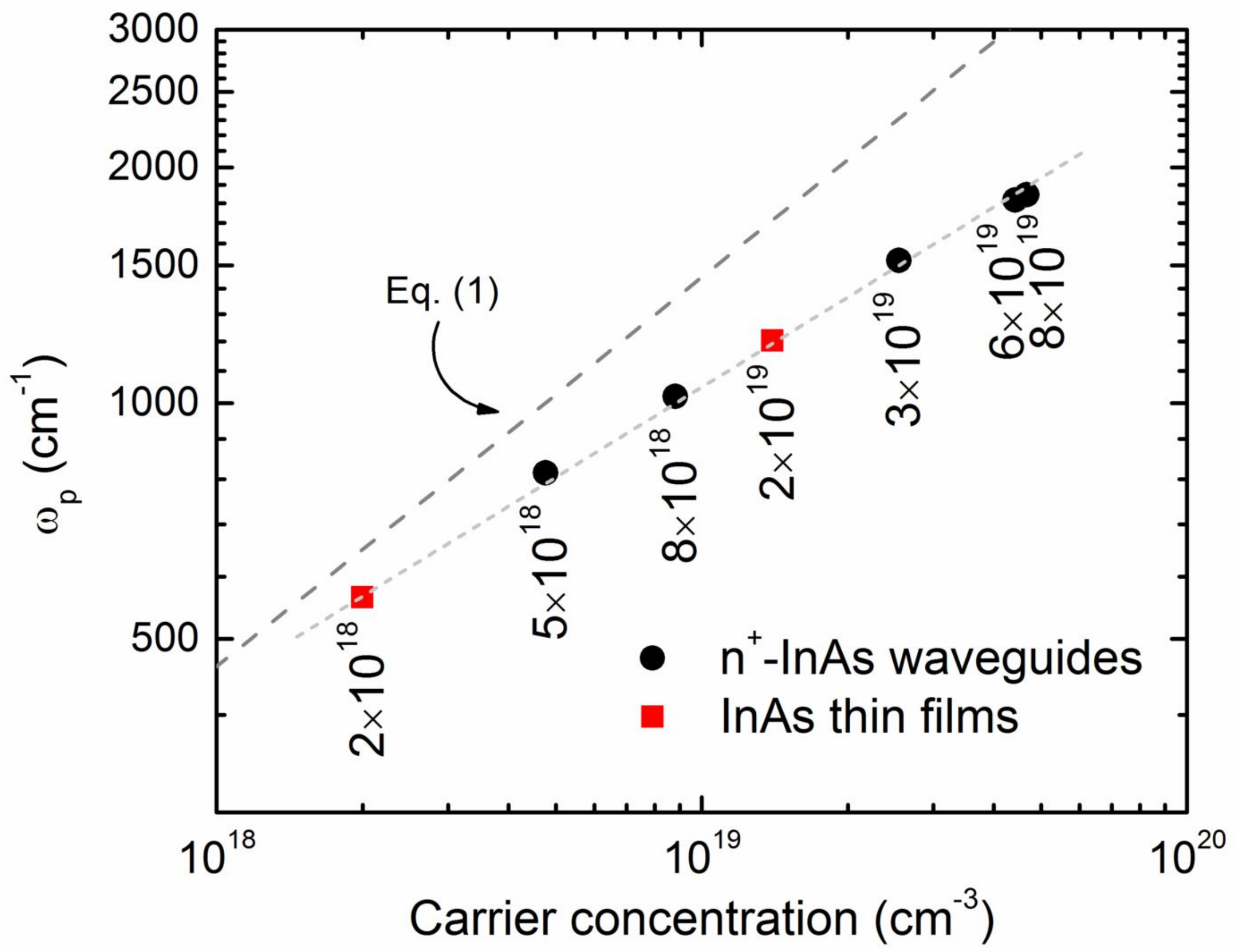




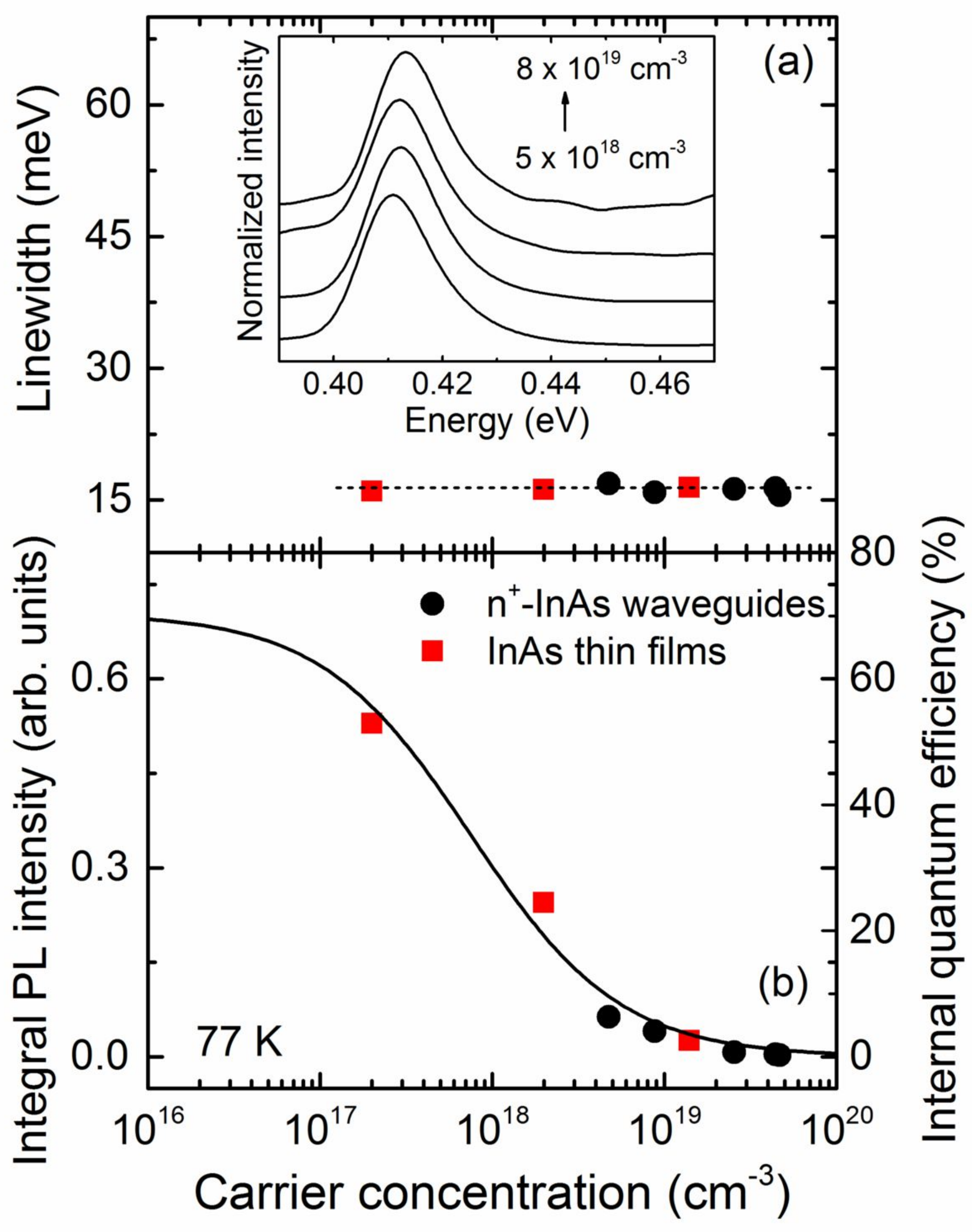

